\section{One-Step, Highly Efficient Site-Directed Mutagenesis by Toxic Protein Selection}

BioTechniques 32:1266-1270 (June 2002)

\section{ABSTRACT}

A fast and efficient site-directed mutagenesis method has been developed, using the newly constructed plasmid pTPS19, which expresses the toxic $C c d B$ protein originally encoded by the E. coli $F$ plasmid. Once the target gene is cloned into pTPS19, desired mutations can be introduced with two primers. The first contains the desired mutation, and the second is designed to create a +1 frame shift in the $\mathrm{ccdB}$ gene to inactivate the $C c d B$ protein. The mutants can be directly selected on LB plates containing IPTG, through which the toxic $C c d B$ protein is induced, thereby eliminating cells carrying wild-type parental plasmids. Based on stringent selection through the toxic $C c d B$ protein, mutagenesis efficiency of 90\%-100\% was reached even after one round of transformation.

\section{INTRODUCTION}

Site-directed mutagenesis provides a powerful tool to study gene expression and protein structure-function relationships. The basic principle of the method involves enzymatic extension of an oligonucleotide primer containing desired mutations in a target gene cloned into a single-stranded phage vector or a double-stranded plasmid vector, followed by subsequent selection against wild-type parental templates (7). Several strategies have been described to improve mutagenesis efficiency, mainly through modification of the parental template or the newly synthesized mutant strand by differential methylation (10), uracil incorporation (2), thionucleotide incorporation (14), or other novel modifications $(4,5,8,11)$.

Here we describe a highly efficient site-directed mutagenesis system that depends on toxic protein selection (TPS). By using TPS mutagenesis, we successfully created many mutants, in- cluding greater than 15 random mutants of the human Sam68 ( Src-asssociated protein in mitosis), to identify dominant negative mutants of this protein (15). TPS was also used either to delete a 120bp fragment encoding the $\mathrm{KH}$ domain of Sam68 or to insert a 36-bp DNA fragment into a codon-optimized HIV-1 gp160 construct to correct an unexpected deletion error generated during PCR.

\section{MATERIALS AND METHODS}

\section{Construction of pTPS19}

We constructed plasmid pTPS19 from a zero-background cloning vector, pZErO-2.1 (Invitrogen, Carlsbad, CA, USA), by deleting the original multiple cloning site (MCS) (nucleotides 274370) and part of the lacZ $\alpha$ gene (nucleotides 395-562); then a new MCS (a 323-bp PvuII fragment) from pUC19 was inserted into the unique $B s p H I$ restriction site (nucleotide 2453) of pZErO-2.1 to yield pTPS19 (Figure 1).

The toxic $c c d B$ gene in pTPS19 was originally carried by the $E$. coli F plasmid. F plasmid contains two genes, $c c d A$ and $c c d B$, which contribute to plasmid stability by killing progeny cells that have not inherited a plasmid copy at cell division (9). $c c d B$ encodes a potent cytotoxin of 101 amino acids and acts by enhancing ATP-dependent DNA cleavage by DNA gyrase (3). TPS mutagenesis takes advantage of the stringent selection of $\mathrm{CcdB}$ against wild-type parental plasmid, which cannot coexist in the same cells along with mutant plasmids, even after a single round of transformation, thereby eliminating the need for a second round of transformation.

\section{Procedure of TPS Mutagenesis}

TPS mutagenesis was performed after target genes had been cloned into the MCS of pTPS19. The polymerization reaction was carried out according to a modified unique site elimination mutagenesis (5). In $20 \mu \mathrm{L}, 100 \mathrm{ng}$ plasmid, $450 \mathrm{ng}$ mutagenic primer, and $150 \mathrm{ng}$ selection primer were incubated in annealing buffer $(20 \mathrm{mM}$ Tris- $\mathrm{HCl}, \mathrm{pH}$ $7.5,10 \mathrm{mM} \mathrm{MgCl}, 50 \mathrm{mM} \mathrm{NaCl})$ at $104^{\circ} \mathrm{C}$ (in oil bath) for $4 \mathrm{~min}$ and placed immediately on ice for $5 \mathrm{~min}$. Three microliters of $10 \times$ synthesis buffer $(100$ $\mathrm{mM}$ Tris- $\mathrm{HCl}, \mathrm{pH} 7.5,5 \mathrm{mM}$ dNTPs, $20 \mathrm{mM}$ DTT) and $3 \mu \mathrm{L} 10 \mathrm{mM}$ ATP (to

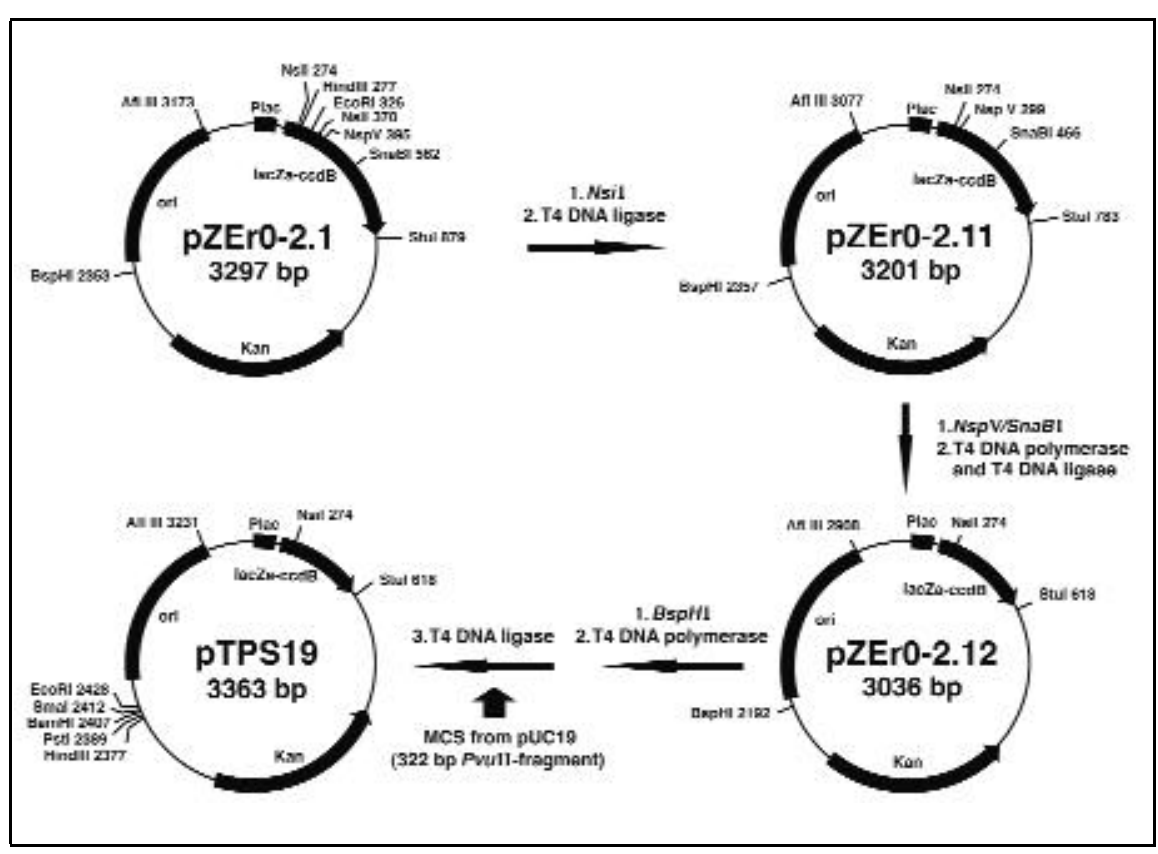

Figure 1. Construction of pTPS19. The starting plasmid pZErO-2.1 was digested by NsiI to remove the MCS (96 bp). Then, a 165-bp Nsp V-SnaBI fragment from the lacZa gene was also deleted to derive plasmid pZErO-2.12. Plasmid pZErO-2.12 was linearized by $B s p H I$, and blunt ends were introduced with $\mathrm{T}_{4}$ DNA polymerase; the fragment was then ligated with a blunt-ended MCS from pUC19, a 323-bp PvuII fragment, to construct plasmid pTPS19. 
Table 1. Oligonucleotide Primers for 15 Random Sam68 Mutants

\begin{tabular}{|ccc|}
\hline $\begin{array}{c}\text { Primer } \\
\text { Name }\end{array}$ & Position & Primer Sequencea $\left.\mathbf{( 5}^{\prime} \rightarrow \mathbf{3}^{\prime}\right)$ \\
\hline PM1 & $1117-1152$ & GCCCGGGCCTCGCACCGGTCGCAGCCGCCACCG \\
PM2 & $1225-1259$ & GCCACAGCCTCGGACCGGTTGGAGCCAGAGAAC \\
PM3 & $1282-1314$ & GAGAAGGACTCGCACCGGTCGTCCTTCACTCAC \\
PM4 & $1444-1475$ & GTCAAGCAGTATCACCGGTTCAATTTTTGG \\
PM5 & $1556-1587$ & GGGCTCAATGAGAGACCGGTCCAAGGAGGAAGAG \\
PM6 & $1662-1693$ & CCCCCATGTGAGGACCGGTCTCTTATGGCCCATG \\
PM7 & $1824-1855$ & GGCCGGGGAGCTGACCGGTCTCCACCACCTGTTC \\
PM8 & $1878-1909$ & CCACCTCGGGGGGACCGGTACGTGGTACACCAG \\
PM9 & $1932-1963$ & GGTGCCACTGTGAACCGGTGCGTGCCACCCCCAC \\
PM10 & $1984-2016$ & GCACCAAGAGCACACCGGTCGGGCATCCAGAGG \\
PM11 & $2043-2070$ & CCAGAAACATATGACCGGTATGGATATGATG \\
PM12 & $2094-2121$ & TACGAAGGCTACGACCGGTATTACAGCCAG \\
PM13 & $2146-2179$ & GACTATGGACATGACCGGTTTCAAGATTCTTATG \\
PM14 & $2199-2233$ & CTGGAATGGGACCAACCGGTCGCTGAAGGCCCCTC \\
PM15 & $2254-2286$ & GCATACAGAGAGCACCGGTATGGACGTTATT \\
aUnderlined bases represent the Agel sites introduced. \\
\hline
\end{tabular}

a final concentration of $1 \mathrm{mM}$ ) were then added to the $20 \mu \mathrm{L}$ annealed DNA mixture with $1 \mu \mathrm{L} \mathrm{T}_{4}$ DNA polymerase (3 U/ $\mu \mathrm{L}$, New England Biolabs, Beverly, MA, USA), $1 \mu \mathrm{L} \mathrm{T}_{4}$ DNA ligase (3 U/ $\mu L$; Promega, Madison, WI, USA), and $2 \mu \mathrm{L}$ water. The reaction mixture was incubated at $37^{\circ} \mathrm{C}$ for $2 \mathrm{~h}$. $\mathrm{T}_{4}$ DNA polymerase and $\mathrm{T}_{4}$ DNA ligase were inactivated by heating the reaction mixture to $68^{\circ} \mathrm{C}$ for $10 \mathrm{~min}$ before transformation of competent E. coli BMH71-18 cells (BD Biosciences Clontech, Palo Alto, CA, USA).

Competent BMH71-18 cells (100 $\mu \mathrm{L}$ ) were transformed with $10 \mu \mathrm{L}$ of the reaction mixture by heating the cell/DNA mixture at $42^{\circ} \mathrm{C}$ for $1 \mathrm{~min}$. One milliliter of LB medium was then added to the cell/DNA mixture and incubated at $37^{\circ} \mathrm{C}$ with shaking for $1 \mathrm{~h}$. Then, final concentrations of $50 \mu \mathrm{g} / \mathrm{mL}$ kanamycin and $2 \mathrm{mM}$ IPTG were added, and the culture was incubated at 


\section{Short Technical Reports}

$37^{\circ} \mathrm{C}$ for $1 \mathrm{~h}$ before plating onto LB agar plates supplemented with $50 \mu \mathrm{g} / \mathrm{mL}$ kanamycin, $50 \mu \mathrm{g} / \mathrm{mL}$ tetracycline, and $2 \mathrm{mM}$ IPTG. After incubating at $37^{\circ} \mathrm{C}$ for 8-9 h (can be extended to overnight incubation), colonies were randomly selected and transferred to LB medium containing $50 \mu \mathrm{g} / \mathrm{mL}$ kanamycin and grown at $37^{\circ} \mathrm{C}$ for plasmid isolation.

\section{RESULTS AND DISCUSSION}

\section{High Efficiency of TPS Mutagenesis}

To investigate the feasibility of TPS and determine its mutagenesis efficiency, we started directly from pTPS19 and designed primers to create an additional PstI restriction site at position 2553 in pTPS19. The mutagenic primer for the target mutation was 5'-CGTT CACTGACET GCA GACCCCGGAA$3^{\prime}$ (synthesized by Invitrogen), which changes GT to TG (in bold; PstI restriction site is underlined). The selection primer was 5'-GTACGGCATTAAA GTTTACACCTATA-3', which is almost identical to the $5^{\prime}$ sequence of $c c d B$ coding region (nucleotides 301327 in pTPS19) but contains an insertion (underlined) to create $\mathrm{a}+1$ frame shift in $c c d B$. The selection primer is versatile and was used for all mutagenesis experiments described.

After TPS mutagenesis, 20 colonies were randomly selected for plasmid minipreps. The target mutation introduced an additional PstI recognition site at position 2557 in pTPS19, which contained another PstI site at position 2389. Therefore, by Pst I digestion, the mutant plasmid was cut into two fragments of 169 and 3194 bp. However, the wild-type parental pTPS 19 was linearized into a 3363-bp fragment. We found that the mutagenesis efficiency of the desired mutation varied from $90 \%$ to $100 \%$ when the ratio of mutagenic primer:selection primer changed from $1: 1$ to $3: 1$ (data not shown). The 3:1 mutagenic primer:selection primer ratio proved to be high enough to ensure sufficient binding and priming of the mutagenic primers.

\section{Generation of Sam68 Mutants by TPS Mutagenesis}

TPS mutagenesis was performed to create mutations of several target genes, including the human Sam68 gene. Sam68 cDNA (1.7 kb) was subcloned into pTPS19 to yield pTPS-Sam68, which was grown in JM109 cells. Fifteen mutagenic primers (PM1-PM15) were synthesized (Invitrogen), in which an extra AgeI recognition site was introduced (Table 1). Since there is a natural AgeI within the Sam68 gene, all the desired mutant plasmids should be cut into two fragments by AgeI, but wild-type parental plasmid only yielded a single band of $5.1 \mathrm{~kb}$ (Figure 2). We analyzed five colonies for each mutagenesis reaction; mostly, all five of these were mutants. Rarely, only four out of five colonies contained the desired muta-

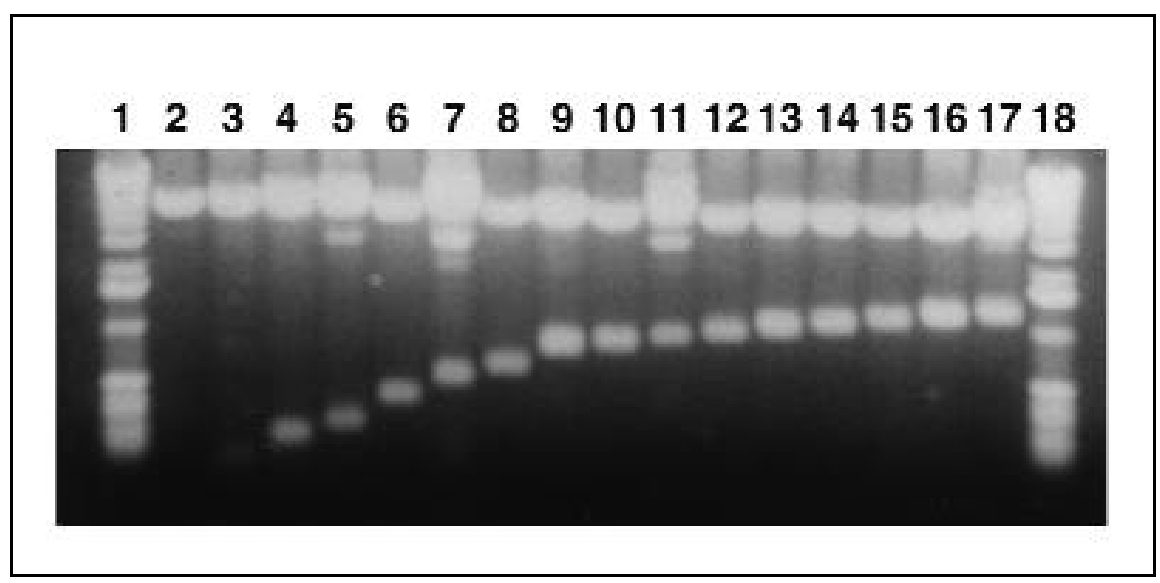

Figure 2. Generation of 15 random mutants of Sam68. Fifteen random point mutations were confirmed by AgeI restriction analysis. Lanes 1 and 18, DNA marker containing 1-kb ladder (Invitrogen); lane 2, wild-type pTPS-Sam68 linearized by AgeI; lanes 3-17, random mutants of PM1-PM15 digested by AgeI. In lanes 5, 7, and 11, a 3-kb band appeared because of partial AgeI digestion. tion. All Sam68 mutants were tested for their interaction with wild-type Sam68 and HIV-1 Rev protein. Interestingly, mutant PM15 (P439R) was a dominant negative mutant that inhibited Sam68 and HIV-1 Rev function (13).

We also generated a Sam68 mutant with a deletion in the central $\mathrm{KH}$ domain (12). This $\Delta \mathrm{KH}$ mutant was shown previously to inhibit wild-type Sam68 function during the G1/S transition in the cell cycle (1). However, $\Delta \mathrm{KH}$ did not inhibit Sam68 to substitute HIV Rev function (12).

\section{Correction of HIV-1 gp160 Con- struct by TPS Mutagenesis}

HIV-1 has a biased codon usage that differs from codon usage for human genes. The expression level of HIV-1 envelope was significantly higher after codon-optimization, using the codons preferred by human cells (6). We have codon-optimized the envelope gene of a Zambian pediatric primary isolate, 1084i (16), with 52 overlapping oligonucleotide primers (about $100 \mathrm{bp}$ each). After annealing these primers, the full-length gene was amplified using the high-fidelity PCR system (Roche Applied Science, Indianapolis, IN, USA). Sequencing revealed that the synthetic gene contained several point mutations and a 36-bp deletion at the 3 '-end. TPS mutagenesis was employed to correct the PCR errors. The corrected, optimized HIV-1 1084i env was confirmed by DNA sequence analysis and transient transfection. Functional envelope glycoproteins were expressed (data not shown).

To summarize, TPS mutagenesis could efficiently introduce point mutations, deletions, and insertions. It takes advantage of a stringent selection against wild-type parental plasmid through the action of $\mathrm{CcdB}$ protein that is toxic to $E$. coli. The mutagenesis process was also considerably shortened. As for many other mutagenesis methods, one of the disadvantages for TPS mutagenesis is that the target gene needs to be subcloned into pTPS19 first. However, once the target gene is subcloned, the resultant plasmid can be repetitively used as the template to generate different mutants later on. In the meantime, the same selection primer may be shared by all 


\section{Short Technical Reports}

different mutagenesis reactions. We conclude that the high fidelity, low background, and the relatively short hands-on time make TPS a useful alternative mutagenesis procedure.

\section{REFERENCES}

1.Barlet, I., F. Maurier, M. Duchesne, E. Guitard, B. Tocque, and F. Schweighoffer. 1997. A role for Sam68 in cell cycle progression antagonized by a spliced variant within the KH domain. J. Biol. Chem. 272:31293132.

2.Bauer, C.E., S.D. Hesse, D.A. WaechterBrulla, S.P. Lynn, R.J. Gumport, and J.F. Gardner. 1985. A genetic enrichment for mutations constructed by oligodeoxynucleotidedirected mutagenesis. Gene 37:73-81.

3.Bernard, P., K.E. Kézdy, L.V. Melderen, J. Steyaert, L. Vyns, M.L. Pato, P.N. Higgins, and M. Couturier. 1993. The F plasmid CcdB protein induces efficient ATP-dependent DNA cleavage by gyrase. J. Mol. Biol. 234:534-541.

4.Boren, J., I. Lee, M.J. Callow, E.M. Rubin, and T.L. Innerarity. 1996. A simple and efficient method for making site-directed mutants, deletions, and fusions of large DNA such as P1 and BAC clones. Genome Res. 6:1123-1130.

5.Deng, W.P. and J.A. Nickoloff. 1992. Site-directed mutagenesis of virtually any plasmid by eliminating a unique site. Anal. Biochem. 200:81-88.

6.Haas, J., E.C. Park, and B. Seed. 1996 Codon usage limitation in the expression of HIV-1 envelope glycoprotein. Curr. Biol. 6:315-324

7.Hutchison, C.A., S. Phillips, M.H. Edgell, S. Gillam, and M. Smith. 1978. Mutagenesis at a specific position in a DNA sequence. J. Biol. Chem. 253:6551-6560.

8.Inouye, S. and M. Inouye. 1987. Oligonucleotide-directed site-specific mutagenesis using double-stranded plasmid DNA, p. 181206. In S.A. Narang (Ed.), Synthesis and Application of DNA and RNA. Academic Press, New York.

9.Jaffé, A., T. Ogura, and S. Hiraga. 1985. Effects of the ccd function of the F plasmid on bacterial growth. J. Biol. Chem. 163:841-849.

10.Kunkel, T.A. 1985. Rapid and efficient sitespecific mutagenesis without phenotypic selection. Proc. Natl. Acad. Sci. USA 82:488492.

11.Lewis, M.K. and D.V. Thompson. 1990. Efficient site directed in vitro mutagenesis using ampicillin selection. Nucleic Acids Res. 18:3439-3443

12.Reddy, T.R., W. Xu, J.K. Mau, C.D. Goodwin, M. Suhasini, H. Tang, K. Frimpong, D.W. Rose, and F. Wong-Staal. 1999. Inhibition of HIV replication by dominant negative mutants of Sam68, a functional homolog of HIV-1 Rev. Nat. Med. 5:635-642.

13. Reddy, T.R. 2000. A single point mutation in the nuclear localization domain of Sam68 blocks the Rev/RRE-mediated transactivation.
Oncogene 19:3110-3114.

14.Taylor, J.W., J. Ott, and F. Eckstein. 1985. The rapid generation of oligonucleotide-directed mutations at high frequency using phosphorothioate-modified DNA. Nucleic Acids Res. 13:8765-8785.

15.Taylor, S.J. and D. Shalloway. 1994. An RNA-binding protein associated with $\mathrm{Src}$ through its $\mathrm{SH} 2$ and $\mathrm{SH} 3$ domains in mitosis. Nature 368:867-871.

16.Xu, W., B.A. Smith-Franklin, P.L. Li, C Wood, J. He, Q. Du, G.J. Bhat, C. Kankasa, et al. 2001. Potent neutralization of primary human immunodeficiency virus clade $\mathrm{C}$ isolates with a synergistic combination of human monoclonal antibodies raised against clade $\mathrm{B}$. J. Hum. Virol. 4:55-61.

This work was supported by National Institutes of Health grant no. 19972 to B.A.M., GM56089 to F.W.-S., AI46240 to T.R.R., and P01AI48240 to R.M.R. The authors thank Susan Sharp for her assistance in the preparation of this manuscript. Address correspondence to Dr. Ruth $M$. Ruprecht, Dana-Farber Cancer Institute, 44 Binney Street, Boston, MA 02115, USA. email: ruth_ruprecht@dfci.harvard.edu

Received 20 November 2001; accepted 17 January 2002.

W. Xu ${ }^{1,2,3}$, Y. Zhang1, L.-Y. Yeh$^{1}$, C.R. Ruprecht ${ }^{1}$, F. WongStaal $^{2}$, B.A. McFadden ${ }^{3}$, T.R. Reddy $^{4}$, and R.M. Ruprecht ${ }^{1}$ 'Dana-Farber Cancer Institute

Boston, MA

${ }^{2}$ University of California at San Diego

La Jolla, CA

${ }^{3}$ Washington State University

Pullman, WA

${ }^{4}$ Wayne State University

Detroit, MI, USA

For reprints of this or any other article, contact Reprints@BioTechniques.com

\section{Minimal FLAG Sequence Useful in the Functional Epitope Tagging of $\mathrm{H}$-Ras}

BioTechniques 32:1270-1280 (June 2002)

\section{ABSTRACT}

Epitope tagging can interfere with normal protein function, indicating the need for an unobtrusive epitope tag. The FLAG ${ }^{\circledR}$ epitope (DYKDDDDK) was examined for a minimal epitope useful in the tagging of $\mathrm{H}$ Ras. The heptapeptide tag, F7 (MDYKD $D D)$, was found to retain reactivity with $M 2$ and M5 monoclonal antibodies in immunoprecipitation, Western blotting, and immunofluorescence microscopy. The F7 tag did not interfere with Ras stability, EGF stimulation of Ras activation, and downstream phosphorylation of MAPK Erk1/2. Unlike the full FLAG sequence, the F7 tag had minimal effect on the growth properties of H-Ras in a colony-forming assay. The F7 tag may be useful when minimizing the effect of tagging on protein function is an important criterion in the selection of an $\mathrm{N}$-terminal epitope tag.

\section{INTRODUCTION}

A common approach to studying a specific cellular protein is to transfect the gene of interest into cultured mam malian cells and examine functional properties of the expressed protein. The incorporation of an epitope tag on the protein provides a convenient handle for isolating and studying the exogenous protein. In principle, any short oligopeptide may serve as an epitope tag. How ever, oligopeptides that serve as efficient immunogens are more practical for obtaining peptide-specific monoclonal antibodies of high affinity (6). In addition, the epitope tag must localize to the surface of the attached protein to be accessible to antibodies and should not interfere with the normal function of the target protein. Occasionally, protein tags alter the conformation and function of the fusion protein. For instance, the 11amino-acid substance $\mathrm{P}$ tag has been shown to interfere with the normal structure of intermediate filament proteins (2). In the study of the small G-protein Ras, the GST and His tags were found to 